\title{
Identification of brassinosteroid genes in Brachypodium distachyon
}

\author{
Claudia Corvalán ${ }^{1}$ and Sunghwa Choe $e^{1,2,3^{*}}$ (B)
}

\begin{abstract}
Background: Brassinosteroids (BRs) are steroidal phytohormones that are involved in diverse physiological processes and affect many important traits, such as plant stature, stress tolerance, leaf angle, fertility, and grain filling. BR signaling and biosynthetic pathways have been studied in various plants, such as the model dicot Arabidopsis thaliana; however, relatively little is known about these pathways in monocots.

Results: To characterize BR-related processes in the model grass Brachypodium distachyon, we studied the response of these plants to the specific BR biosynthesis inhibitor, propiconazole (Pcz). We found that treatments with Pcz produced a dwarf phenotype in B. distachyon seedlings, similar to that observed in Pcz-treated Arabidopsis plants and in characterized BR-deficient mutants. Through bioinformatics analysis, we identified a list of putative homologs of genes known to be involved in BR biosynthesis and signaling in Arabidopsis, such as DWF4, BR6OX2, CPD, BRI1, and BIN2. Evaluating the response of these genes to PCZ treatments revealed that candidates for BdDWF4, BR6OX2 and, CPD were under feedback regulation. In addition, Arabidopsis plants heterologously expressing BdDWF4 displayed tall statures and elongated petioles, as would be expected in plants with elevated levels of BRs. Moreover, heterologous expression of $B d B I N 2$ in Arabidopsis resulted in dwarfism, suggesting that BdBIN2 functions as a negative regulator of BR signaling. However, the dwarf phenotypes of Arabidopsis bri1-5, a weak BRI1 mutant allele, were not complemented by overexpression of $B d B R / 1$, indicating that BdBRI1 and BRI1 are not functionally equivalent.

Conclusion: We identified components of the BR biosynthetic and signaling pathways in Brachypodium, and provided examples of both similarities and differences in the BR biology of these two plants. Our results suggest a framework for understanding BR biology in monocot crop plants such as Zea mays (maize) and Oryza sativa (rice).
\end{abstract}

Keywords: BIN2, Brachypodium distachyon, Brassinosteroids, BRI1, DWF4, Monocots, Propiconazole

\section{Background}

Brassinosteroids (BRs) are plant polyhydroxylated steroids that function as growth-promoting hormones. They have been implicated in many developmental and physiological processes in Arabidopsis thaliana, including vascular differentiation, stem and root elongation, reproductive development, photomorphogenesis, and stress responses. Hence, mutant plants defective in BR synthesis or perception display characteristic phenotypes such us short stature, round, curled leaves, short petioles, and reduce fertility [1-3].

\footnotetext{
* Correspondence: shchoe@snu.ac.kr

${ }^{1}$ School of Biological Sciences, College of Natural Sciences, Seoul National University, Seoul 08826, South Korea

${ }^{2}$ Convergence Research Lab for Plant Functional Products, Advanced Institutes of Convergence Technology, Suwon 16229, Gyeonggi-do, South Korea

Full list of author information is available at the end of the article
}

The BR-biosynthetic and signaling pathways have been well studied in Arabidopsis thaliana. A family of enzymes belonging to Cytochrome P450s mediates most of the steps of BR biosynthesis, and characterization of mutants defective in these enzymes contributed to the understanding of BR biology $[4,5]$. Various approaches have established the components and mechanisms of the $B R$ signaling pathway; once BR binds to the receptor kinase, BRASSINOSTEROID-INSENSITIVE 1 (BRI1), transmits the signal to downstream genes, eventually leading to the repression or activation of BR responsive genes $[6,7]$.

It appears that BR signaling is conserved between monocotyledonous and dicotyledonous plants, as some counterparts of the Arabidopsis proteins are present in Oryza sativa (rice). For instance, orthologs of the receptor BRI1 and co-receptor BRI1-ASSOCIATED RECEPTOR KINASE 
1 (BAK1), named OsBRI1 and OsBAK1, respectively; two homologs of the negative regulator of the BR signaling protein BR INSENSITIVE 2 (BIN2), GSK3/SHAGGY-like kinase 1 and 2 (OsGSK1 and OsGSK2); and an ortholog of a major transcription factor in the BR transduction pathway BRASSINAZOLE RESISTANT 1 (OsBZR1) have been identified in rice [7-10].

Despite the similarities with dicot BR biology, some differences have been noted in monocots too. Brassinolide (BL), the most active form of BR and end product of $B R$ synthesis in Arabidopsis, has not been detected in rice, where apparently castasterone (CS) seems to be the end product. Furthermore, no homolog of CYP85A2, which mediates BL synthesis, has been found in rice [11]. In addition, components of BR signaling with no known orthologs in Arabidopsis have been identified in rice, indicating the existence of specific BR functions in monocots or some degree of functional redundancy. Examples of these components are DWARF AND LOW-TILLERING (DTL) and TILLER ANGLE INCREASED CONTROLLER (LIC), which act downstream of OsBRI1 and OsGSK2 to positively and negatively regulate rice $\mathrm{BR}$ signaling, respectively $[12,13]$, and the U3 ubiquitin ligase TAIHU DWARF 1 (TUD1), which interacts genetically and physically with D1/OsRGA, a heterotrimeric G protein subunit involved in gibberellin (GA) and BR responses [14].

Brachypodium distachyon (hereafter Brachypodium) is a relatively new model plant proposed for the study of grasses, since it has a short live cycle, is self-fertile, easy to grow, and is more closely related to Poaceae than is $A$. thaliana [15]. However, little is known about phytohormones in B. distachyon. Only two dwarf mutants, with defects in the BRI1 receptor and a C-6 oxidase (BRASSINOSTEROID DEFICIENT DWARF 1; BRD1), have been characterized in this model plant $[16,17]$.

In the present work, we used a specific BR biosynthesis inhibitor drug, propiconazole (Pcz) [18], to study BR action in Brachypodium and characterize orthologs of the BR-biosynthetic enzymes DWARF4, BR6ox2, and CPD. Furthermore, by heterologous complementation, we studied homologs of two important genes in the BR signaling pathway, the receptor BRI1 and the negative regulator BIN2. This work revealed important similarities and differences between the BR synthesis and signaling pathways in Arabidopsis and Brachypodium.

\section{Results}

\section{Brachypodium distachyon seedlings display BR-related} phenotypes in response to propiconazole treatment

Since Brachypodium is a relatively new model plant, studies of processes and genes regulated by phytohormones in this organism are limited. The unavailability of Brachypodium BR-defective or -insensitive mutants made it challenging to determine if $\mathrm{BR}$ function is conserved across plant species. To study the mode of action of BRs and the factors involved in these processes, we first treated Brachypodium Bd21 seedlings with the BR-specific inhibitor Pcz in concentrations ranging from 1 to $50 \mu \mathrm{M}$ for 7 days (Fig. 1A-C). We observed a dose-response reduction of the total lengths of plants; $1 \mu \mathrm{M}$ Pcz resulted in a $\sim 13 \%$ reduction in length compared to mock conditions, whereas $50 \mu \mathrm{M}$, the greatest concentration tested, resulted in a $\sim 60 \%$ reduction (Fig. $1 \mathrm{~A}$ ). This reduction was especially severe in roots; the main root was reduced by $50 \%$ in plants treated with $20 \mu \mathrm{M}$ Pcz relative to control plants, and by over $74 \%$ in those treated with $50 \mu \mathrm{M}$ (Fig. 1B). The Pcz-induced inhibitory effects were observed under both light and dark conditions (Additional file 1: Figure S1). In contrast to the effect on the overall length of the plant, leaf length was only reduced by $\sim 25 \%$ under the strongest Pcz treatment (Additional file 2: Figure S2). To evaluate if Pcz had other effects on leaf morphology or the vascular system, we examined the leaf architecture in more detail, focusing on venation patterns, total number of veins, vein density, and distance between veins. Interestingly, we found that Pcz-treated leaves were thicker and wider than those from plants grown under control conditions, but that the number of veins remained the same. As a consequence, the distance between veins is on average greater in the treated leaves, so vein density is reduced by Pcz treatment (Fig. 2).

\section{Identification of genes modulating BR response in Brachypodium}

With the release of the whole genome sequence of Brachypodium distachyon, we were able to search for homologs that could participate in brassinosteroid biosynthesis or signaling in this species. We conducted a BLAST search of the Brachypodium database using the amino acid sequences of Arabidopsis proteins and then used multiple sequence alignment (MSA) and phylogenetic analysis to reduce the number of homo$\log$ candidates to one per protein for further evaluation. In the case of BIN2, the MSA and phylogenetic analysis were performed using Arabidopsis BIN2, BIN2-LIKE 1 (BIL1), and BIN2-LIKE 2 (BIL2) and also the rice homologs OsGSK1, OsGSK2, and OsSKetha. We also performed a second MSA, including A. thaliana BIN2, a GSK3 from human (P49841) and D. melanogaster (P18431), and rice OsKetha (Y13437) using the T-Coffee program to screen for conserved motifs. We observed that the kinase domain and the TREE domain, identified as being a putative Thr phosphorylation site by caseine kinase II and thus important for negative regulatory events, were also present in the Brachypodium homolog (Fig. 3). Thus, we selected Bradi2g48280, Bradi2g32620, Bradi2g36370, Bradilg23550, Bradi1g69040, Bradi4g43110, 

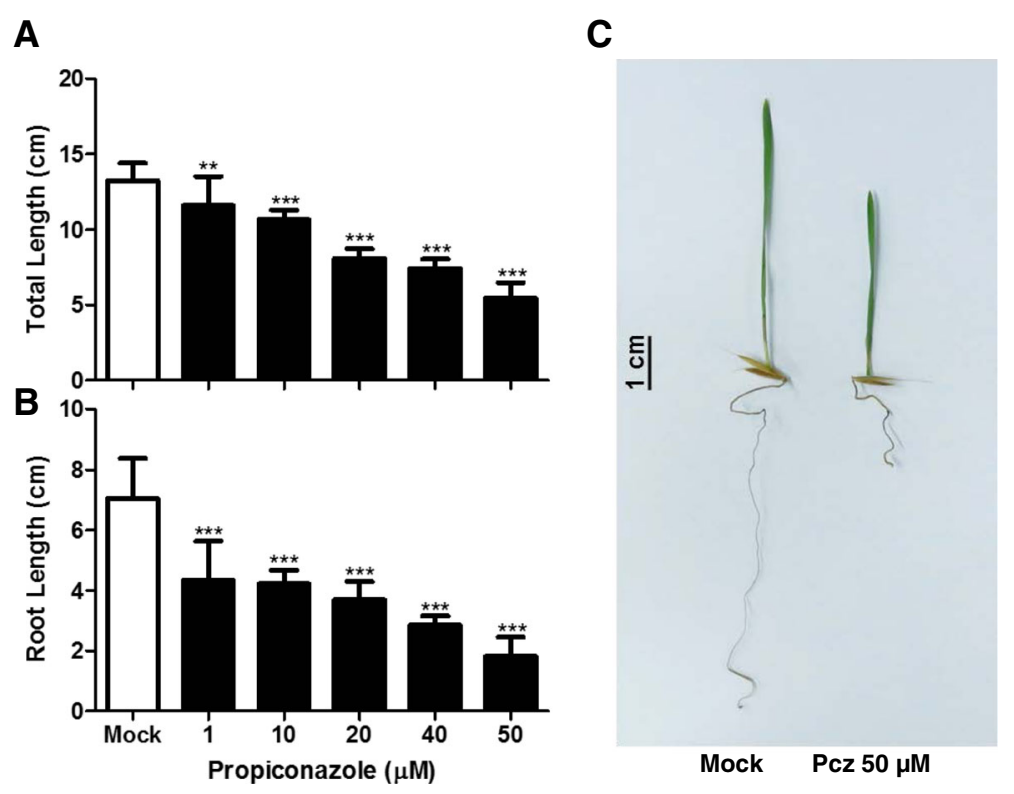

Fig. 1 Brachypodium seedlings exhibit dose-dependent dwarfism in response to propiconazole treatment. a Total lengths of seedlings and (b) roots after 7 days of exposure to 0 (Mock) to 50 MM Pcz. c Morphology of 7-days-old seedlings subjected to the mock treatment and the strongest Pcz concentration tested. The graphs represent average value $(n>10)$ and error bars standard deviation. Significant differences among treatments were determined by Student's t-test. ${ }^{* *}, P<0.001$ and ${ }^{* *}, P<0.0001$

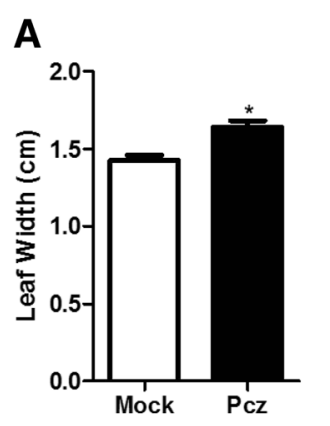

B
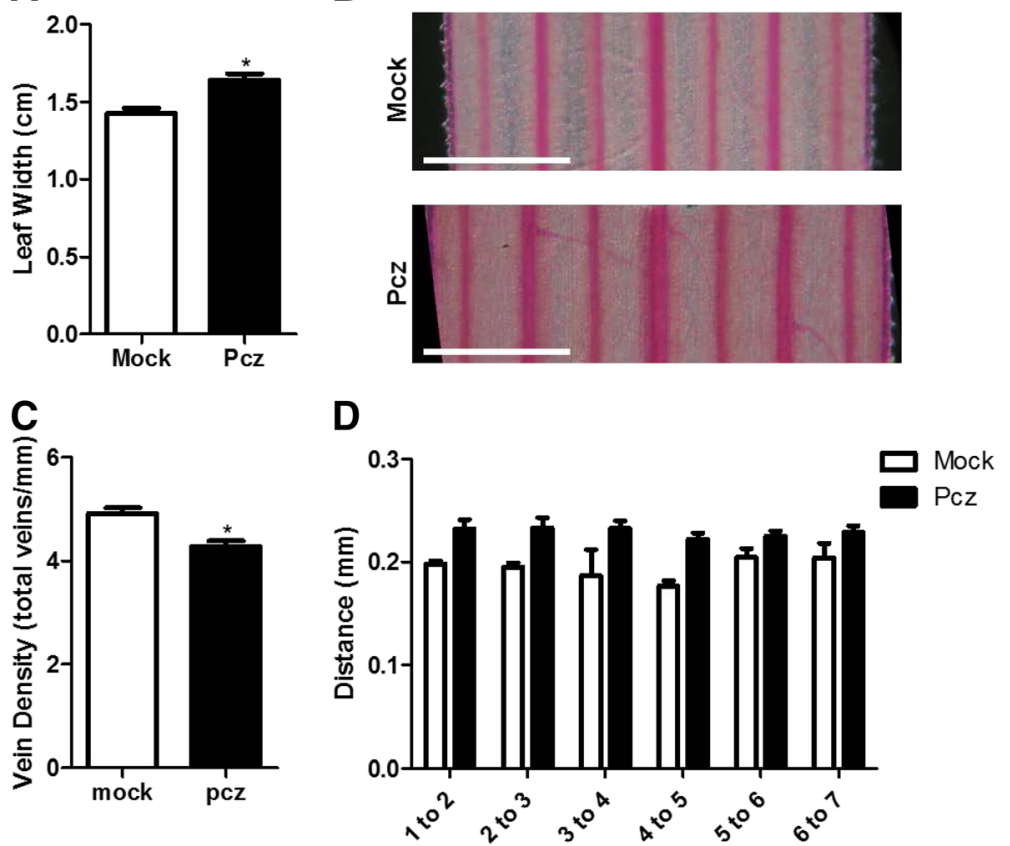

Fig. 2 Brachypodium leaf width and distance between veins are affected by Pcz. a Leaves of seedlings undergoing mock or Pcz (50 $\mu M)$ treatments were measured and dissected in the middle. $\mathbf{b}$ Safranin O-stained leaf sections were used to count and observe veins under the microscope. $\mathbf{c}$ The vein density values were obtained by dividing the total number of veins in the section by the width of the leaf. $\mathbf{d}$ The distance between veins was measured using ImageJ software. Scale bar $=0.5 \mathrm{~mm}$. The graphs represent average value $(n>5)$ and error bars standard deviation. Significant differences among treatments were determined by Student's t-test. *, $P<0.05$ 


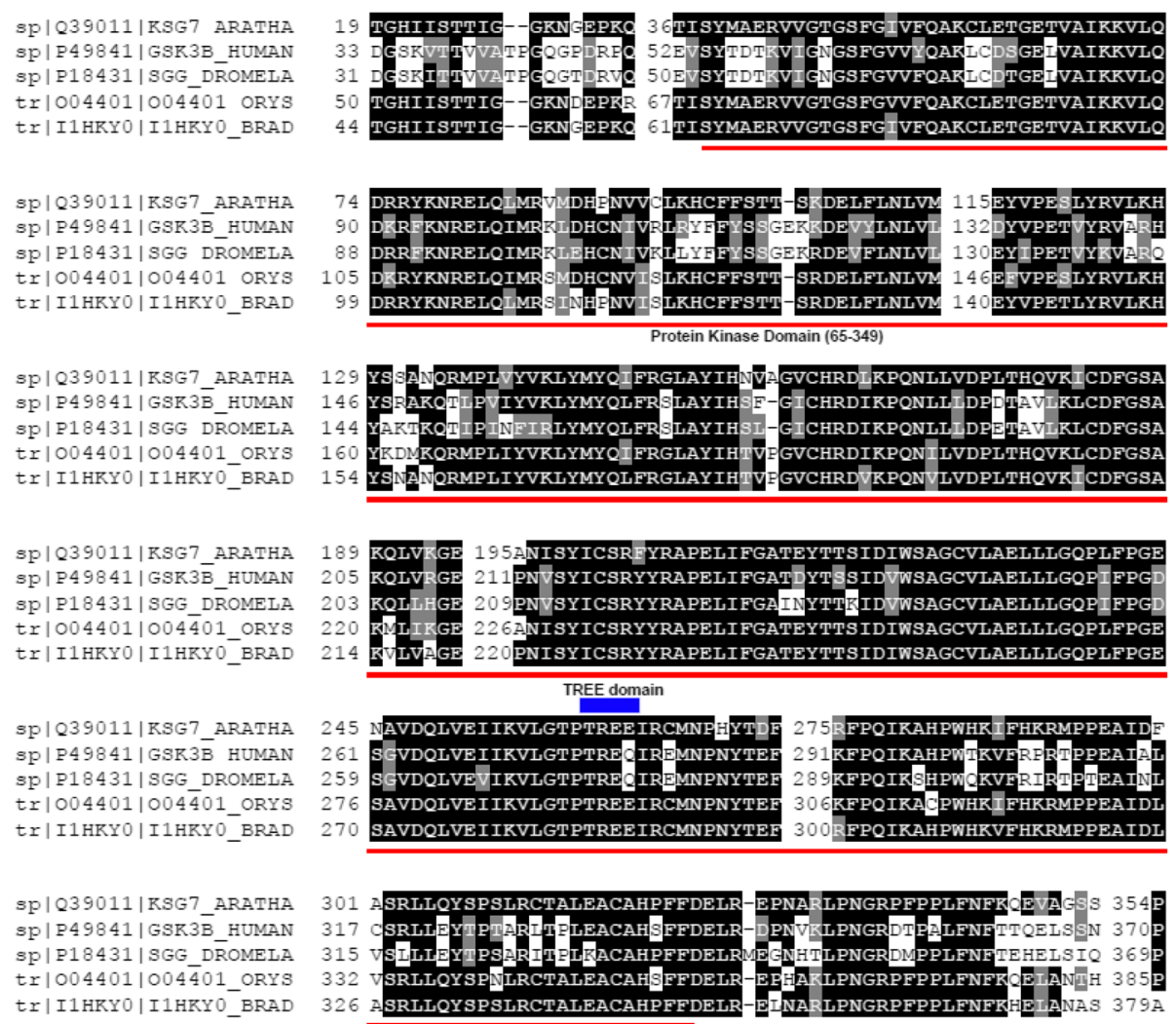

Fig. 3 BdBIN2 shows conserved motifs with other GSK3/SHAGGY-like kinase proteins. Part of the multiple sequence alignment (MSA) of the BIN2 ortholog in Brachypodium (1HKY0) with AtBIN2 (Q39011) and a GSK3 homolog from human (P49841) and D. melanogaster (P18431). The conserved protein kinase domain is underlined in red and the TREE domain, which is well conserved in plants, is represented by a blue rectangle

and Bradi1g15030 as homologs of BRI1, BIN2, BSU1, $B Z R 1, D W F 4, C P D$, and $B R 6 o x 2$, respectively, for further characterization.

\section{Brachypodium DWF4, CPD, and BR6OX2 homologs are under feedback regulation}

To verify that the genes identified by bioinformatics tools were in effect orthologs of BR biosynthetic genes in $B$. distachyon, we examined their transcripts levels in seedlings subjected to various Pcz treatments. To obtain a general sense of the gene expression response to the treatments, we performed semi-quantitative RT-PCR analysis on seedlings treated with three different concentrations of Pcz $(10,25$, and $50 \mu \mathrm{M})$ along with mock. We noticed that the transcript levels of the three BR-related genes increased with increasing Pcz concentrations. The GA biosynthesis gene GA20ox1 was used as a control for the Pcz treatment, to ensure that only BR-related genes were inhibited by the treatment (Fig. 4A). After determining the conditions that produced a clear transcriptional response, we carried out a similar experiment using just mock and one Pcz concentration $(50 \mu \mathrm{M})$ to analyze $B d D W F 4, B d B R 6 \circ x 2$, and $B d C P D$ mRNA levels by quantitative real-time RT-PCR. For the first two genes, Pcz-treated seedlings exhibited a more than sixfold increase in expression with respect to the mock treatment, while $B d C P D$ was only slightly upregulated (Fig. 4B-D). Nonetheless all three candidate genes were found to be under negative feedback regulation, a characteristic of genes involved in BR biosynthesis. Normally, the expression of these genes is inhibited by the presence of BR and enhanced when BR levels are low such as under treatment with inhibitors (i.e., a negative feedback mechanism regulates the activity of genes downstream of BR). Thus, Bradi1g69040 (BdDWF4), Bradi4g43110 (BdCPD), and Bradi1g15030 (BR6ox2) may indeed be involved in BR biosynthesis in Brachypodium distachyon.

\section{Overexpression of BdDWF4 in Arabidopsis thaliana produce long and slender plants}

To further evaluate the involvement of BdDWF4 in BR responses, we introduced the full-length coding sequence of this gene into $A$. thaliana under the CaMV 35 s promoter. From a total of 26 analyzed BdDWF4 overexpressing lines (BdDWF4ox), half displayed an increase in plant height and had longer and narrower leaves than the wild-type control, Col-0 (Fig. 5A). These characteristics are typical of brassinosteroid-overproducing mutant plants, such as 


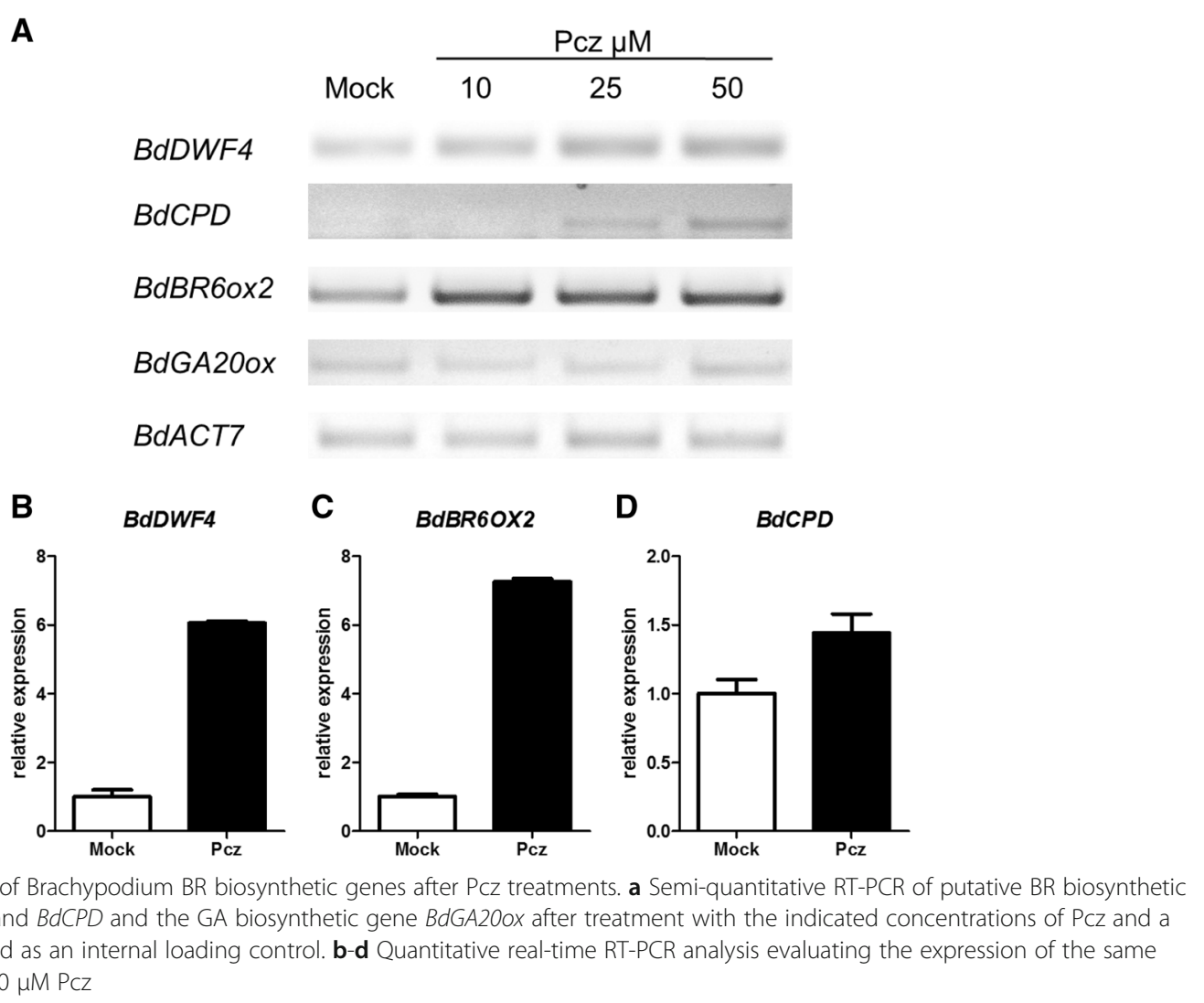

gulliver3 - $D$ (gul3-D), in which the increase in BR level is the result of activation tagging of DWF4. Analysis of the expression level in transgenic plants corroborated that degree of the phenotype is proportional to the levels of transcripts (Fig. 5C). The results strongly suggest that Bradilg69040 plays a significant role in Brachypodium growth and development, probably by functioning in the BR biosynthetic pathway.

\section{Overexpression of BdBIN2 in Arabidopsis results in a stunted growth phenotype}

We then overexpressed a candidate gene involved in BR signaling BdBIN2, in A. thaliana to study the effect of this gene on the phenotypes of the transgenic plants. From a total of 32 analyzed lines, nearly $14 \%$ of the transgenic plants presented severe dwarf phenotypes, were unable to set flowers, and died after a few weeks (Fig. 5B). These extreme dwarf plants presented the highest level of transcript accumulation (Fig. 5D). Other BdBIN2ox lines showed milder phenotypes that were able to produce seeds but still exhibited a compact stature and smaller curled leaves compared with the wild type (Fig. 5B). These findings insinuate that Bradi2g32620 is a functional ortholog of BRIN2 in
Brachypodium that operates as a negative regulator of the BR signaling pathway.

\section{$B d B R I 1$ overexpression does not revert the weak phenotype of $A$. thaliana bri1-5}

A search for BRI1 in the Brachypodium genome yielded three candidate genes (Additional file 3: Table S1) that we arranged in a phylogenetic tree along with other BRI1 proteins described in various dicot (A. thaliana, pea (Pisum sativum), and tomato (Lycopersicon esculentum)) and monocot (rice (Oryza sativa) and barley (Hordeum vulgare)) plants. As a reference, we also used BRI1-LIKE proteins BRL1, BRL2, and BRL3 from Arabidopsis and rice. As shown in Figure 6A, we observed that the protein product of Bradi2g48280 was in the same group as orthologs of BRI1 in monocots, and although it shares only $\sim 53 \%$ amino acid sequence identity with AtBRI1, it shares more than $80 \%$ identity with amino acid sequences in its monocot counterparts. To test if Bradi2g48280 (named BdBRI1 from now on) has the same functions as AtBRI1, we performed heterologous complementation experiments. Specifically, we examined if overexpression of BdBRI1 was able to revert the dwarf phenotype of the BR-insensitive bri1-5 mutant, which is a weak allele of AtBRI1. In a first experiment we obtained 

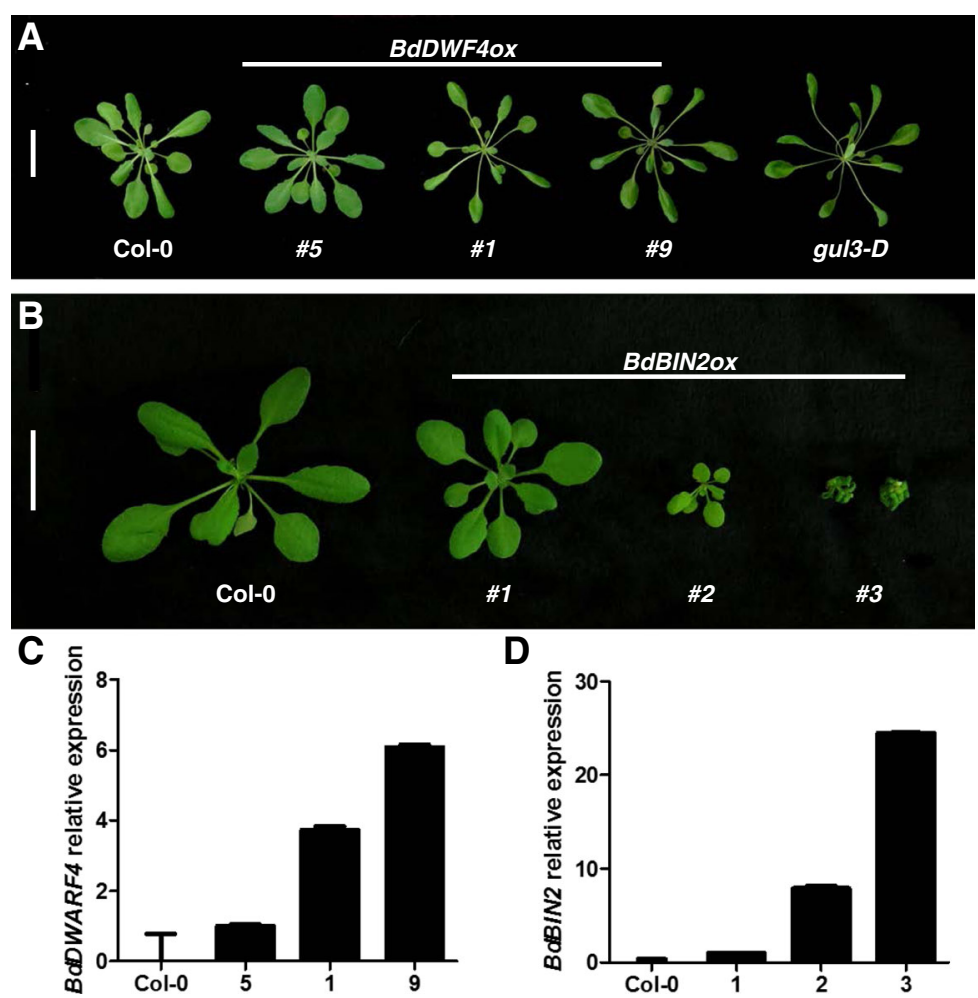

Fig. 5 Phenotypes of the BdDWF4Ox and BdBIN2ox transgenic lines, suggesting the involvement of BdDWF4 in BR biosynthesis and BdBIN2 in responses, respectively. a Morphology of three independent BdDWF4 overexpression lines (\#5, \#1, and \#9) along with wild type Col-0 and gul3-D BR overproducing mutant. b Phenotypes of the wild type Col-0 and BdBIN2 overexpressing lines. Line \#2 represent mild phenotype, while \#3 are examples of severe dwarfism. Scale bar $=2 \mathrm{cms}$. c-d Quantitative real-time analysis evaluating the heterologous expression of BdDWF4 and BdBIN2 respectively. Line \#5 c and \#1 (d) which don't show distinctive phenotype from wild type were used to compare expression of the rest of the lines. AtUBQ10 was used as internal loading control

13 independent lines, all exhibiting dwarf phenotypes similar to bri1-5. Similarly, in a second transformation we obtained another 21 transgenic lines resistant to the Basta which didn't show rescued phenotypes (Additional file 4: Figure S3A); bri1-5/BdBRI1ox lines were slightly taller than the bri1-5 control, but were still dwarfed with abnormal leaf morphology (Fig. 6B-C). These results indicate that although Bradi2g48280 is likely a homolog of Arabidopsis thaliana BRI1, these two genes may have different structure and/or functions that make BdBRI1 incapable of operating as the BR receptor in Arabidopsis.

\section{Discussion}

The use of knock-out and activation tagging mutants has facilitated the characterization of many important genes involved in phytohormone signaling and biosynthetic pathways. In the case of BRs, the characterization of dwarf mutants mainly in Arabidopsis has contributed to our current understanding of BR biology. However, few studies have evaluated BRs in Poaceae, and most advances in these crops have been made in rice and maize [18-21]. The lack of BR-defective mutants is an obstacle in efforts to reveal BR functions in other model plants and at the time this work started, just one BR mutant had been identified in Brachypodium [16]. Subsequently, one more mutant defective in BR biosynthesis has emerged [17].

In this report, we sought to contribute to the knowledge of BR biology using Brachypodium as a model plant by measuring the effects of a potent and specific BR inhibitor, Pcz. We demonstrated that Pcz treatments mimic the characteristic dwarf phenotypes of plants deficient in brassinosteroids and that the response is dose-dependent, similar to what was described for Arabidopsis and maize [18]. Treatments with $50 \mu \mathrm{M} \mathrm{Pcz}$ caused severe phenotypes in seedlings, especially in the roots, where we observed a $75 \%$ decrease in length compared to the mock treatment, while the reduction in leaf length was almost $34 \%$. The marked effect on roots may be due to the method used to apply Pcz. As we planted the seedlings in vermiculite soil soaked with Pcz solution, the roots were in permanent and direct contact with Pcz, whereas the aerial tissue was not. Still, this method was able to cause not only a decrease in leaf 

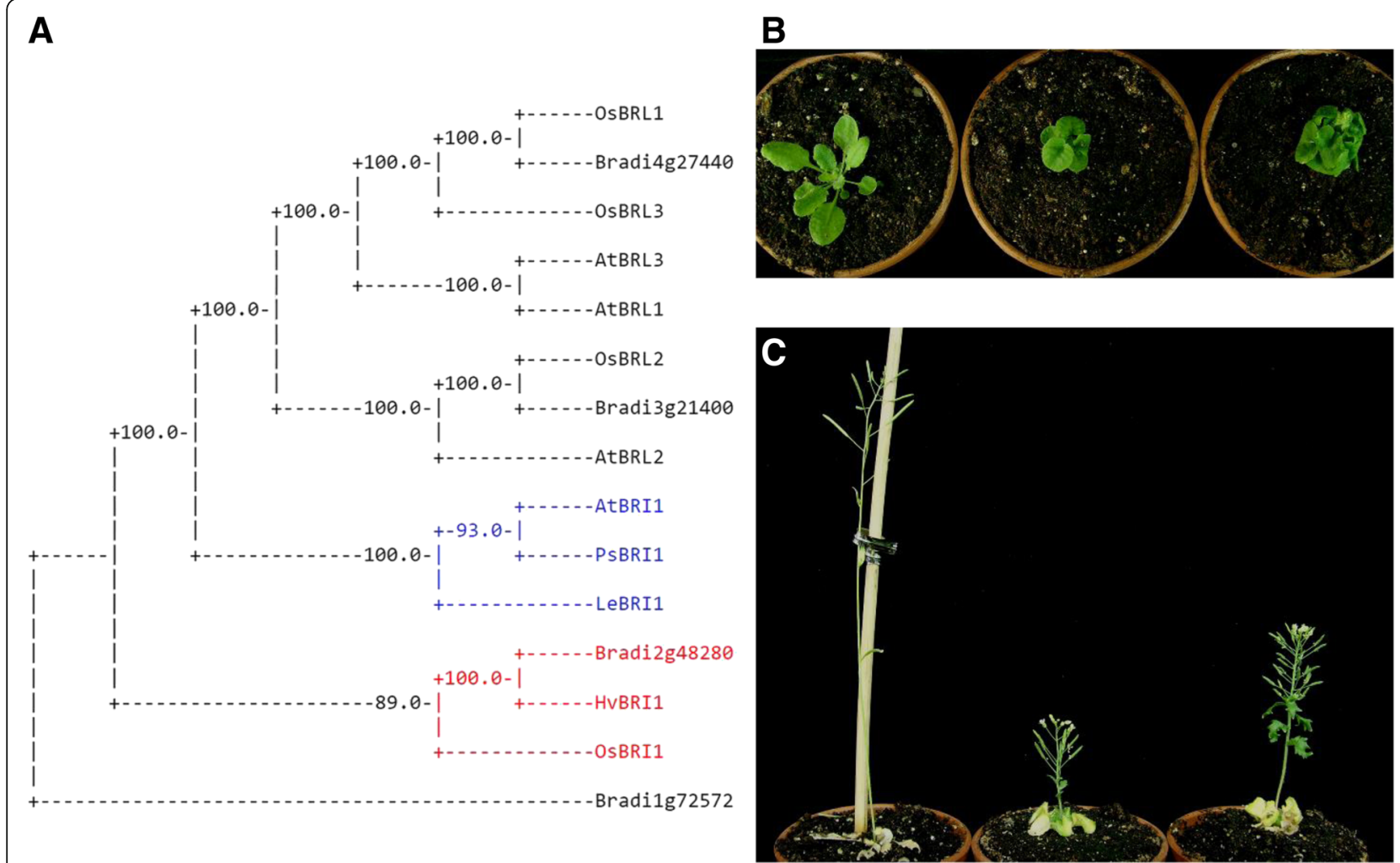

Fig. 6 BR/1 homolog in Brachypodium fails to rescue the phenotypes of the BR-insensitive mutant bri1-5. a Phylogenetic analysis showing Brachypodium BRI1 candidate proteins and BRI1 homologs in other species, including Arabidopsis, tomato, pea, rice, and barley. b Phenotypes of 3-week- or (c) 6-week-old WS-2 wild-type adult plants, the bri1-5 mutant, and transgenic bri1-5/BdBR/10x plants

length, but also other phenotypes in the leaves that were similar to the ones described in transgenic rice plants overexpressing the BR negative regulator BIN2 [10].

Although the Pcz-treated leaves initially seemed to develop relatively normally, they later curled, especially towards the top, and exhibited increased width and thickness compared to the mock treatments. Further analyses using cross-sections of leaves could reveal more about the possible effects of $\mathrm{Pcz}$ on venation pattern or cell organization in these tissues. Considering the phenotypes obtained using the chemical inhibitor in Brachypodium, we propose that Pcz treatments represent a powerful tool for studying BRs in this model plant. Pcz could be administered to investigate vascular and stomatal development, root growth, cell elongation, and meristematic cell maintenance, among other specific processes in which BR is known to play active roles [22, 23].

The BR biosynthetic genes are primarily regulated at the transcriptional level, and this regulation is mainly mediated by the transcription factor BZR1, with supplemental regulation by other transcription factors. When BR signaling is activated, the dephosphorylated (i.e., active) form of BZR1 binds to the promotor sequence of BR synthetic genes to repress their transcription. This mechanism is known as negative feedback downregulation of BR biosynthetic genes $[4,24]$. In this way, BL treatments reduce the expression of DWF4, CPD, and BR6ox2 while Pcz application has the opposite effect since it reduce BR levels [18]. Considering this feedback regulation of the biosynthetic genes, we evaluated the transcript levels of our candidate BR-related genes BdDWF4 (Bradi1g69040), BdCPD (Bradi4g43110), and BdBR6ox (Bradi1g15030) in seedlings subjected to Pcz treatment. We observed the Pcz-dependent induction of the three candidates tested, although the increase in $B d C P D$ expression level was lower than that of the other two genes. However, a similar result was observed previously for Arabidopsis $C P D$ upon Pcz application [18], possibly because another mechanism regulates the post-transcriptional level of $C P D$. Taken together, BdDWF4, BdCPD, and BdBR6ox are regulated by a feedback loop at the transcript level once BR levels decrease upon Pcz treatments, suggesting their possible role as enzymes involved in BR synthesis.

To date, many BR genes homologs to the ones characterized from $A$. thaliana have been identified in different monocot species and most of these works are based on the description of the phenotypes from mutant plants which in most of cases present dwarfism or other characteristic BR-related phenotypes. Nevertheless, not many of these studies examine the effects of the ectopic 
overexpression of these suggested homolog genes in Arabidopsis to examine if they fulfill similar roles to its dicot equivalent. Some exceptions are the overexpression of maize DWF4 (ZmDWF4) and rice GSK1, one of the homologs of BIN2, in Arabidopsis [25, 26]. Ectopic overexpression of ZmDWF4 generated Arabidopsis plants with longer petioles, narrower leaves, and increased size [25], which is similar to the effects we obtained by overexpressing BdDWF4. This typical growth-promoting phenotype is also portrayed by transgenic plants overexpressing AtDWF4 [27] and the gain-of-function mutant gulliver3 - D/dwarf4 - D, which we used as a control to compare the effects of our overexpression [28]. In case of BIN2, opposite phenotypes are observed due to its role in modulating BZR1/BES1 degradation. Heterologous expression of the rice BIN2 homolog OsGSK1 in Arabidopsis resulted in plants that were barely one-third the height of control plants [26]. Similarly, overexpression of BdBIN2 affected plant height and other features in our experiments, but to a greater extent. This difference may be due to OsGSK1 having a minor role in BR signaling, since another homolog of BIN2 in rice, OsGSK2, seems to be a more prominent negative regulator that functions upstream of transcription factors such as OsBZR1, LIC, and DTL [10].

Homologs of the BR receptor BRI1 have been identified and studied in three monocot species to date, including rice, barley, and Brachypodium [29-32]. The functions of the BRI1 homolog in Brachypodium were revealed in studies of plants in which BdBRI1 was silence by RNAi technology [30], while the homologs in the other two species were characterized using mutant plants. In addition to OsBRI1, BRI1-LIKE homologs (OsBRL1 and OsBRL3) have also been characterized in rice, and in both rice and barley different alleles of BRI1 have been found in later works [33, 34]. However, these studies failed to determine if the monocot homologs of BRI1 are able to function as a BR receptor in dicot plants. This fact is indeed important, since the end product of BR synthesis and more active BR in monocots does not seem to be brassinolide, but castasterone. Thus, the structure of BRI1 remained to be compared in monocot and dicot plants [11]. To do so, we first searched the Brachypodium genome for BRI1 putative homologs. We found that Bradi2g48280 likely functions as a BR receptor in Brachypodium [30]; however, to test our hypothesis, we cloned the gene and heterologously expressed it in A. thaliana bri1-5 mutant plants. To our surprise, the full-length coding sequence of $B d B R I 1$ was not able to rescue the dwarf phenotype of bri1-5, which is a weak allele of bri1. This was observed despite the plants were expressing the Brachypodium version of BRI1 (Additional file 4: Figure S3B). Several transgenic lines showed a slight increase in total plant height, but still presented extremely curled leaves and defects in flowering and seed formation. As far as we know, this is the first time a monocot version of BRI1 has been expressed in A. thaliana to evaluate if complementation is possible. Our results represent an early step for comparing the functions and structures of BRI1 between dicot and monocot plants and thus broaden the understanding about BR response in crop plants. BRs have profound effects on several physiological responses such as plant size, fertility and leaves inclination, thus good biotechnological targets to manipulate plant architecture towards desirable agronomic traits. The identification and characterization of BR genes in Brachypodium along with the use of genetic engineering have the potential to enhance crop yield in important crops and even the use of fertilizers could be potentially reduced with less negative environmental effects.

\section{Conclusion}

Our results demonstrated that the use of propiconazole in Brachypodium mimic the dwarf phenotypes of BRdeficient or -insensitive plants, thus Pcz is a potent tool to study BR responses in this model plant. We identified components of the BR biosynthetic pathway DWF4, CPD and BR6ox2, and BRI1, BIN2, BSU1 and BZR1 of the BR signaling pathway in Brachypodium. We showed that $B d D W F 4, B d C P D$, and BdBR6ox are regulated by negative feedback loop at the transcript level and overexpression of BdDWF4 in Arabidopsis conferred transgenic plants with similar phenotypes to those overexpressing Arabidopsis DWF4. We concluded that Bradilg69040, Bradi4g43110, and Bradilg15030 (BdDWF4, BdCPD, and BdBR6ox respectively) encode $B R$ biosynthetic genes with functions similar to their homologs in Arabidopsis. Heterologous expression of Bradi2g32620 (BdBIN2) caused dwarf phenotypes in Arabidopsis. We therefore conclude that Bradi2g32620 likely functions as BR negative regulator in Brachypodium. Furthermore, the Bradi2g48280 (BdBRI1) gene likely functions as a BR receptor in Brachypodium. However, the full-length coding sequence of $B d B R I 1$ was not able to rescue the dwarf phenotype of bri1-5, which is a weak allele of bri1 in Arabidopsis. This results indicate that BdBRI1 and BRI1 may not be functionally equivalent. Further investigations are needed to verify the differences in the BR biology of monocot and dicot plants.

\section{Methods}

\section{Plant material and growth conditions}

Brachypodium distachyon ecotype $\mathrm{Bd} 21$, a community standard diploid inbred line, was used in this research according to previous description in Hong et al. [35]. Seeds were sterilized and germinated in water-soaked filter paper for 3 days before being transferred to soil. Plants were grown in growth room conditions at $22{ }^{\circ} \mathrm{C}$ 
and a long-day (16 h light, $8 \mathrm{~h}$ dark) photoperiod. Arabidopsis thaliana wild-type Col-0 and Ws-2 ecotypes or mutant seeds (gulliver3 - D and bri1-5) were surface-sterilized before being sprinkled on agar-solidified MS (Murashige and Skoog) medium. After three day of stratification at $4{ }^{\circ} \mathrm{C}$, plates were transferred to a growth room and grown under long-day conditions at $22{ }^{\circ} \mathrm{C}$. After 10 days, seedlings were transferred to soil and maintained under the same conditions in a growth room.

\section{Chemical treatments and morphometric analysis}

For all treatments in Brachypodium, 3-day-old seedlings were transferred to coarse vermiculite soaked in the indicated concentrations of propiconazole solution or mock (DMSO) for control plants and grown for an additional 7 days. Seedlings were maintained in a growth room under normal long-day conditions, except for those grown under dark conditions, in which plates were covered with aluminum foil to mimic growth under total darkness. Plants were then harvested, photographed, and analyzed using ImageJ software. Leaf length was measured from the root-shoot transition zone to the tip of the main leaf, whereas the length of the main root was used to determine root length. For leaf width, each leaf was measured in the exact middle to make an appropriate comparison. After measurements were taken, leaves were sectioned in the middle and cleared by washing in ethanol $(\mathrm{EtOH})$ solutions. Sections were first washed in $90 \% \mathrm{EtOH}$ at $37{ }^{\circ} \mathrm{C}$ for $3 \mathrm{~h}$. If necessary, the $90 \%$ solution was replaced to expedite clearing. Then sections were replaced in $50 \%$ solution for 5 minutes.. Once the sections were cleared, they were placed overnight in $1 \%$ Safranin O diluted in $50 \%$ ethanol, to stain the veins a brilliant red color and facilitate observation under a microscope. A Primo Vert Inverted Microscope (Zeiss) was used to examine and image the samples, and then Image software was used to calculate the total width and other parameters, such as distance between veins.

\section{Identification of BR-related genes in Brachypodium}

Using the UniProt server (http://uniprot.org/blast/), Arabidopsis and rice (Oryza sativa) BR-related genes were searched for in the Brachypodium genome by BLAST. For each search, query peptide sequences of well-known Arabidopsis proteins involved in BR signaling, including BRI1, BIN2, BSU1, and BZR1, and synthesis, including DWF4, $\mathrm{CPD}$, and BR6OX2, were used. A multiple sequence alignment (MSA) was performed using the T-Coffee Multiple Sequence Alignment Server (http,//tcoffee.crg.cat/) on the identified sequences with at least $40 \%$ identity and previously characterized proteins with equivalent functions in other plant species. BoxShade software (http,//embnet.vital-it.ch/software/BOX_form.html)

was used to identify and visualize conserved sequences. A Neighbor-joining phylogenetic tree was built using the obtained MSA to illustrate the relatedness among known proteins and homolog candidates in $B$. distachyon and to select one for further characterization. A list of the BR-related proteins and candidates in $B$. distachyon used for the alignments and the MSAs can be found in (Additional file 3: Table S1).

\section{RT-PCR and quantitative real-time RT-PCR}

For gene expression analysis, total RNA was prepared from Mock- or Pcz-treated B. distachyon seedlings using Trizol (Qiagen). RNA $(2 \mu \mathrm{g})$ was reverse-transcribed using M-MLV enzyme (ELPIS), following the manufacturer's instructions, to synthesize cDNA, which was used as a template in PCR reactions. For semi-quantitative RT-PCR, the resulting cDNA samples were diluted $(1,10)$ and equal amounts were used to amplify each gene. The level of gene expression was defined using $B d A C T 7$ as a reference gene. Quantitative real-time RTPCR detection was performed using the Applied Biosystems StepOne Real-Time PCR System and Power SYBR Green PCR Master Mix in combination with designed primers specific for $B d D W F 4, B d B R 60 x 2$, and $B d C P D$ (Additional file 5: Table S2). The reactions were performed in triplicate and the product was quantified by generating standard curves using serially diluted cDNA mix of all of the samples. Relative expression, representing fold change over the mock control, was normalized for each cDNA sample using $B d A C T 7$ as internal control. As a negative control (NC) we used distilled water in the same amount of the samples. In case of RT-PCR, the conditions were optimized until clear band of expected size was observed in the samples and no band was detected in the NC. In case of quantitative RT-PCR, NC was run in triplicate along with samples and normally gave us an undetermined $\mathrm{C}_{\mathrm{T}}$ value or at least higher value than the most diluted sample from our standard curve to prove the accuracy and specificity of our primers in each run. Melting curves for each set of primers were also generated automatically by the StepOne Software (v2.3) from the Applied Biosystems system.

\section{Cloning of BdDWF4, BdBIN2, and BdBRI1}

To express BdDWF4, BdBIN2, and BdBRI1 under the cauliflower mosaic virus $35 \mathrm{~S}$ promoter (35S) in A. thaliana, RNA was extracted and cDNA was synthesized from $B$. distachyon 10-days-old seedlings and specific primers (Additional file 5: Table S2) were used to amplify full-length coding sequences (CDSs) of interest. PCR product was purified and cloned using the Invitrogen Gateway entry vector $\mathrm{pENTR/SD/D-TOPO}$ in combination with the destination pEarleyGate101 (C-YFP-HA) vector. The constructs were transformed into Arabidopsis using conventional Agrobacterium-mediated techniques. For 
$35 S, B d D W F 4$ and $35 S, B d B I N 2$, plants in the Col-0 background were used, whereas for $35 S, B d B R I 1$, the construct was introduced in bri1-5 mutant background. Transgenic seedlings were selected on MS medium supplemented with $50 \mathrm{mg} / \mathrm{L}$ BASTA.

\section{Additional files}

Additional file 1: Figure S1. Brachypodium seedlings exhibit dwarfism in response to propiconazole treatment in dark. Morphology of 7-days-old seedlings subjected to the mock treatment and $50 \mu \mathrm{M}$ Pcz. (PPTX $1022 \mathrm{~kb}$ )

Additional file 2: Figure S2. Propiconazole treatment reduces leaves and roots lengths in Brachypodium seedlings. Total lengths of seedlings, leaves and roots after 7 days of exposure to mock (white bars) or $50 \mu \mathrm{M}$ PCz (black bars). The graphs represent average value $(n>10)$ and error bars standard deviation. Significant differences among treatments were determined by Student's $t$-test.**, $P<0.0001$. (PPTX $45 \mathrm{~kb}$ )

Additional file 3: Table S1. List of BR genes in A. thaliana and homolog candidates in Brachypodium. (DOCX $17 \mathrm{~kb}$ )

Additional file 4: Figure S3. Phenotypes associated with BdBRl/ox lines and $B d B R / 1$ transcript accumulation. (A) Morphology of 5-week-old transgenic plants selected in Basta media along with Ws-2 wild type and bri1-5 mutant as controls. (B) Expression of BdBR/1 in five of the transgenic plants was confirmed through RT-PCR. AtUBQ10 was used as an internal loading control. (PPTX $1026 \mathrm{~kb}$ )

Additional file 5: Table S2. Primers used in the research. (DOCX 14 kb)

\section{Abbreviations}

BAK1: BRI1-ASSOCIATED RECEPTOR KINASE 1; BIN2: BR INSENSITIVE 2; BL: Brassinolide; BR: Brassinosteroid; BR6ox2: BRASSINOSTEROID-6-OXIDASE 2; BRI1: BRASSINOSTEROID-INSENSITIVE 1; BZR1: BRASSINAZOLE RESISTANT 1; CPD: CONSTITUTIVE PHOTOMORPHOGENIC DWARF; CS: Castasterone; DTL: DWARF AND LOW-TILLERING; DWF4: DWARF 4; GSK: GSK3/SHAGGY-like kinase; LIC: TILLER ANGLE INCREASED CONTROLLER; PCZ: Propiconazole; TUD1: TAIHU DWARF 1, GA, gibberellin, BRD1, BRASSINOSTEROID DEFICIENT DWARF 1

\section{Acknowledgments}

Not applicable.

\section{Funding}

This research was supported, in part, by three grants; the first Next-Generation BioGreen21 Program (PJ01104501) grant played a role in design of the study, the second Cooperative Research Program for Agricultural Science and Technology Development (Project No. PJ01168501) of Rural Development Administration grant helped in collection, analysis, and interpretation of the data, and the third National Research Foundation of Korea (NRF) grant funded by the Korea government (MSIP) (No. 2015R1A2A1A10051668) Republic of Korea supported in writing the manuscript.

\section{Availability of data and materials}

All data generated or analyzed during this study are available from the corresponding author on reasonable request.

\section{Authors' contributions}

CC carried out the experiments, analyzed data and wrote the manuscript. As a major advisor, SC designed the overall research, supported the experiments, interpreted the data, and wrote the manuscript. Both authors have read and approved the final version of this manuscript.

\section{Competing interests}

The authors declare that they have no competing interests.

\section{Consent for publication}

Not applicable.
Ethics approval and consent to participate

Not applicable.

\section{Author details}

${ }^{1}$ School of Biological Sciences, College of Natural Sciences, Seoul National University, Seoul 08826, South Korea. ${ }^{2}$ Convergence Research Lab for Plant Functional Products, Advanced Institutes of Convergence Technology, Suwon 16229, Gyeonggi-do, South Korea. ${ }^{3}$ Plant Genomics and Breeding Institute, Seoul National University, Seoul 08826, South Korea.

Received: 25 July 2016 Accepted: 23 December 2016

Published online: 06 January 2017

References

1. Fujioka S, Sakurai A. Brassinosteroids. Nat Prod Rep. 1997;14(1):1-10.

2. Clouse SD, Langford M, McMorris TC. A brassinosteroid-insensitive mutant in Arabidopsis thaliana exhibits multiple defects in growth and development. Plant Physiol. 1996;111(3):671-8.

3. Choe S, Dilkes BP, Fujioka S, Takatsuto S, Sakurai A, Feldmann KA. The DWF4 gene of Arabidopsis encodes a cytochrome P450 that mediates multiple 22alpha-hydroxylation steps in brassinosteroid biosynthesis. Plant Cell. 1998;10(2):231-43.

4. Chung Y, Choe S. The Regulation of Brassinosteroid Biosynthesis in Arabidopsis. Crit Rev Plant Sci. 2013:32(6):396-410.

5. Kwon M, Choe S. Brassinosteroid biosynthesis and dwarf mutants. J Plant Biol. 2005;48(1):1-15.

6. Li J, Jin H. Regulation of brassinosteroid signaling. Trends Plant Sci. 2007;12(1):37-41.

7. Kim TW, Wang ZY. Brassinosteroid signal transduction from receptor kinases to transcription factors. Annu Rev Plant Biol. 2010;61:681-704.

8. Tong $\mathrm{H}, \mathrm{Chu} \mathrm{C}$. Brassinosteroid signaling and application in rice. Journal of genetics and genomics $=$ Yi chuan xue bao. 2012;39(1):3-9.

9. Sakamoto T. Phytohormones and rice crop yield, strategies and opportunities for genetic improvement. Transgenic Res. 2006;15(4):399-404.

10. Tong H, Liu L, Jin Y, Du L, Yin Y, Qian Q, Zhu L, Chu C. DWARF AND LOWTILLERING acts as a direct downstream target of a GSK3/SHAGGY-like kinase to mediate brassinosteroid responses in rice. Plant Cell. 2012;24(6):2562-77.

11. Kim BK, Fujioka S, Takatsuto S, Tsujimoto M, Choe S. Castasterone is a likely end product of brassinosteroid biosynthetic pathway in rice. Biochem Biophys Res Commun. 2008;374(4):614-9.

12. Zhang C, Xu Y, Guo S, Zhu J, Huan Q, Liu H, Wang L, Luo G, Wang X, Chong $K$. Dynamics of brassinosteroid response modulated by negative regulator LIC in rice. PLoS Genet. 2012;8(4):e1002686.

13. Tong $\mathrm{H}$, Jin $Y$, Liu W, Li F, Fang J, Yin Y, Qian Q, Zhu L, Chu C. DWARF AND LOW-TILLERING, a new member of the GRAS family, plays positive roles in brassinosteroid signaling in rice. The Plant journal, for cell and molecular biology. 2009;58(5):803-16.

14. Hu X, Qian Q, Xu T, Zhang Y, Dong G, Gao T, Xie Q, Xue Y. The U-box E3 ubiquitin ligase TUD1 functions with a heterotrimeric $G$ alpha subunit to regulate Brassinosteroid-mediated growth in rice. PLoS Genet. 2013;9(3): e1003391.

15. Girin T, David LC, Chardin C, Sibout R, Krapp A, Ferrario-Mery S, DanielVedele F. Brachypodium, a promising hub between model species and cereals. J Exp Bot. 2014;65(19):5683-96.

16. Thole V, Peraldi A, Worland B, Nicholson P, Doonan JH, Vain P. T-DNA mutagenesis in Brachypodium distachyon. J Exp Bot. 2012;63(2):567-76.

17. Xu Y, Zhang X, Li Q, Cheng Z, Lou H, Ge L, An H. BdBRD1, a brassinosteroid C-6 oxidase homolog in Brachypodium distachyon L., is required for multiple organ development. Plant Physiol Biochem. 2015:86:91-9.

18. Hartwig T, Corvalan C, Best NB, Budka JS, Zhu JY, Choe S, Schulz B. Propiconazole is a specific and accessible brassinosteroid (BR) biosynthesis inhibitor for Arabidopsis and maize. PLoS One. 2012;7(5):e36625.

19. Zhang C, Bai MY, Chong K. Brassinosteroid-mediated regulation of agronomic traits in rice. Plant Cell Rep. 2014;33(5):683-96.

20. Makarevitch I, Thompson A, Muehlbauer GJ, Springer NM. Brd1 gene in maize encodes a brassinosteroid C-6 oxidase. PLoS One. 2012;7(1):e30798.

21. Hartwig T, Chuck GS, Fujioka S, Klempien A, Weizbauer R, Potluri DP, Choe S, Johal GS, Schulz B. Brassinosteroid control of sex determination in maize. Proc Natl Acad Sci U S A. 2011;108(49):19814-9.

22. Singh AP, Savaldi-Goldstein S. Growth control, brassinosteroid activity gets context. J Exp Bot. 2015;66(4):1123-32. 
23. Fabregas N, Cano-Delgado Al. Turning on the microscope turret, a new view for the study of brassinosteroid signaling in plant development. Physiol Plant. 2014;151(2):172-83.

24. Kim HB, Kwon M, Ryu H, Fujioka S, Takatsuto S, Yoshida S, An CS, Lee I, Hwang I, Choe $\mathrm{S}$. The regulation of DWARF4 expression is likely a critical mechanism in maintaining the homeostasis of bioactive brassinosteroids in Arabidopsis. Plant Physiol. 2006;140(2):548-57.

25. Liu TS, Zhang JP, Wang MY, Wang ZY, Li GF, Qu L, Wang GY. Expression and functional analysis of ZmDWF4, an ortholog of Arabidopsis DWF4 from maize (Zea mays L.). Plant Cell Rep. 2007;26(12):2091-9.

26. Koh S, Lee SC, Kim MK, Koh JH, Lee S, An G, Choe S, Kim SR. T-DNA tagged knockout mutation of rice OsGSK1, an orthologue of Arabidopsis BIN2, with enhanced tolerance to various abiotic stresses. Plant Mol Biol. 2007;65(4):453-66.

27. Choe S, Fujioka S, Noguchi T, Takatsuto S, Yoshida S, Feldmann KA. Overexpression of DWARF4 in the brassinosteroid biosynthetic pathway results in increased vegetative growth and seed yield in Arabidopsis. Plant J. 2001;26(6):573-82.

28. Kim B, Fujioka S, Kwon M, Jeon J, Choe S. Arabidopsis brassinosteroidoverproducing gulliver3-D/dwarf4-D mutants exhibit altered responses to jasmonic acid and pathogen. Plant Cell Rep. 2013;32(7):1139-49.

29. Chono M, Honda I, Zeniya H, Yoneyama K, Saisho D, Takeda K, Takatsuto S, Hoshino T, Watanabe Y. A semidwarf phenotype of barley uzu results from a nucleotide substitution in the gene encoding a putative brassinosteroid receptor. Plant Physiol. 2003;133(3):1209-19.

30. Feng Y, Yin Y, Fei S. Down-regulation of BdBRI1, a putative brassinosteroid receptor gene produces a dwarf phenotype with enhanced drought tolerance in Brachypodium distachyon. Plant science, an international journal of experimental plant biology. 2015;234:163-73.

31. Nakamura A, Fujioka S, Sunohara H, Kamiya N, Hong Z, Inukai Y, Miura K, Takatsuto S, Yoshida S, Ueguchi-Tanaka M, et al. The role of OsBRI1 and its homologous genes, OsBRL1 and OsBRL3, in rice. Plant Physiol. 2006;140(2):580-90

32. Yamamuro C, Ihara Y, Wu X, Noguchi T, Fujioka S, Takatsuto S, Ashikari M, Kitano H, Matsuoka M. Loss of function of a rice brassinosteroid insensitive1 homolog prevents internode elongation and bending of the lamina joint. Plant Cell. 2000;12(9):1591-606.

33. Zhao J, Wu C, Yuan S, Yin L, Sun W, Zhao Q, Zhao B, Li X. Kinase activity of OsBRI1 is essential for brassinosteroids to regulate rice growth and development. Plant science, an international journal of experimental plant biology. 2013;199-200:113-20.

34. Gruszka D, Szarejko I, Maluszynski M. New allele of HvBRl1 gene encoding brassinosteroid receptor in barley. J Appl Genet. 2011;52(3):257-68.

35. Hong SY, Lee S, Seo PJ, Yang MS, Park CM. Identification and molecular characterization of a Brachypodium distachyon GIGANTEA gene, functional conservation in monocot and dicot plants. Plant Mol Biol. 2010;72(4-5):485-97.

\section{Submit your next manuscript to BioMed Central and we will help you at every step:}

- We accept pre-submission inquiries

- Our selector tool helps you to find the most relevant journal

- We provide round the clock customer support

- Convenient online submission

- Thorough peer review

- Inclusion in PubMed and all major indexing services

- Maximum visibility for your research

Submit your manuscript at www.biomedcentral.com/submit

) Biomed Central 